\title{
Gastrointestinal tract involvement in granulomatosis with polyangiitis
}

\author{
Anna Masiak ${ }^{1}$, Łukasz Zdrojewski ${ }^{2}$, Zbigniew Zdrojewski ${ }^{1}$, Barbara Bułło-Piontecka ${ }^{2}$, Bolesław Rutkowski ${ }^{2}$ \\ ${ }^{1}$ Department and Clinic of Internal Medicine, Connective Tissue Diseases and Geriatrics, Medical University of Gdansk, Gdansk, Poland \\ 2Department and Clinic of Nephrology, Transplantology, and Internal Medicine, Medical University of Gdansk, Gdansk, Poland
}

Gastroenterology Rev 2016; 11 (4): 270-275

DOI: $10.5114 / p g .2016 .57887$

Key words: granulomatosis with polyangiitis, gastrointestinal tract bleeding.

Address for correspondence: Anna Masiak MD, PhD, Department and Clinic of Internal Medicine, Connective Tissue Diseases and Geriatrics, Medical University of Gdansk, 7 Dębinki St, 80-001 Gdansk, Poland, phone: +48 5834928 31, +48 606 882 312, e-mail: anna.masiak@wp.pl

\begin{abstract}
Introduction: Granulomatosis with polyangiitis (GPA) is a necrotising vasculitis of small arteries and veins. In its classical manifestation GPA affects the upper and lower respiratory tract and kidneys. However, other organs, including those of the gastrointestinal tract, may be affected as well.

Aim: To present the clinical manifestations of gastrointestinal tract involvement in patients with GPA.

Material and methods: We analysed case records of 34 patients with GPA treated in the Department of Nephrology, Transplantology, and Internal Medicine of the Medical University of Gdansk from 1991 to 2009.

Results: In 9 of 34 patients, 2 men and 7 women, aged 18 to 74 years, gastrointestinal complications were observed in the course of GPA. In two of them a localised and in seven a generalised type of GPA was diagnosed. The main symptoms relating to gastrointestinal tract were: oral mucosa ulcerations, gum mucosa hypertrophy, dyspepsia, vomiting, stomachache, gastrointestinal haemorrhage, diarrhoea, and symptoms of gastrointestinal tract perforation. Two patients required urgent surgical treatment. In 2 of the 5 patients who developed gastrointestinal bleeding, it was the direct cause of death. The histopathological confirmation of specificity of changes in gastrointestinal tract was established only in 2 cases. Tissue samples collected during endoscopy usually revealed only nonspecific inflammation or the presence of ulcers.

Conclusions: Therapeutic strategies accepted for GPA treatment are effective in treating patients with gastrointestinal involvement in the course of the disease. Some complications require surgical intervention.
\end{abstract}

\section{Introduction}

Granulomatosis with polyangiitis (previously Wegener's granulomatosis) (GPA) is a primary vasculitis of unknown origin characterised by granulomatous necrotising inflammation of small arteries and veins. The Chapel Hill Consensus classified GPA as small-vessel vasculitis with the presence of anti-neutrophil cytoplasmic antibodies (CANCA) [1]. Although the classical manifestation of the disease described by Wegener in 1936 [2] includes upper and lower respiratory tract involvement and glomerulonephritis, it can affect every other organ. This results in heterogeneity of clinical manifestations. Although gastrointestinal tract involvement is rare, both the small and the large bowel may be involved, thus causing a serious complication of GPA.

\section{Aim}

The aim of the study was to present the clinical manifestations of gastrointestinal tract involvement in patients with GPA.

\section{Material and methods}

We studied 34 patients with GPA treated in the Department of Nephrology, Transplantology, and Internal Medicine of the Medical University of Gdansk, from 1991 to 2009. Nine (26\%) patients presented symptoms suggesting gastrointestinal tract involvement in the course of GPA. These were 2 men and 7 women, aged 18 to 74 years. The main signs and symptoms are summarised in Table I. The diagnosis of GPA in those patients was established on clinical examination, laboratory, immunological, and histopathological tests, im- 
ages, and endoscopy studies. In 2 patients a localised type of GPA was diagnosed, involving upper airways (patient 4 and 8) and parotid gland (patient 8). In seven other patients a generalised GPA manifested with renal involvement. The division to localised and generalised GPA was based on the European Vasculitis Study Group criteria [3]. Five patients with renal involvement developed kidney function impairment (serum creatinine concentration from 1.9 to $7.8 \mathrm{mg} / \mathrm{dl}$ ). One patient had already been dialysed when GPA was diagnosed (patient 7). In all patients upper respiratory tract involvement was present. In every patient with generalised disease the lower respiratory tract was also involved.

Determination of ANCA was performed by indirect immunofluorescence in a clinical laboratory using our own preparations of neutrophils taken from healthy blood donors, group 0 (1991 to 1994), and commercially available kits (ImmunoConcept 1994 to 2006, and EUROIMMUN Polska since 2006), according to the manufacturer's instructions. Direct ELISAs for PR3-ANCA (ANCA against proteinase-3) were performed with commercially available kits (Varelisa 1991 to 2004, Orgentec 2004 to 2005, Biosystem 2005 to 2007, and INOVA 2007 to 2010).

Eight of 9 patients were anti-neutrophil cytoplasmic antibodies (CANCA) positive. The patient with negative cANCA had localised GPA with symptoms of upper respiratory tract involvement, and the diagnosis was confirmed by the presence of typical granuloma tissue histopathology of the nasal cavity tumour (patient 4). Neutral serine protease (PR3), which is a constituent of azurophil granules of neutrophils, has been identified as the target antigen for c-ANCA in ELISA tests in 6 patients. In 2 cases the test was not performed, another patient with negative ANCA was also antiPR3 negative.
Histopathological confirmation of the disease was obtained in 8 patients. In one patient diagnosis was established on the basis of a typical triad of symptoms and the presence of ANCA only. The results of laboratory and histopathological examinations are summarised in Table II. As a rule, the procedure of induction therapy consisted of methylprednisolone intravenously (0.5$1.0 \mathrm{~g} /$ day for 3-5 days) followed by oral prednisone and cyclophosphamide $\left(0.75 \mathrm{~g} / \mathrm{m}^{2}\right)$ intravenously once a month.

\section{Results}

In 9 of 34 patients gastrointestinal complications were observed in the course of GPA. The most common symptoms were abdominal pain (6 of 9 patients) and gastrointestinal bleeding ( 5 of 9 patients). The other complaints were dyspepsia described as discomfort in the upper abdomen with nausea and loss of appetite (4 of 9), vomiting (2 of 9), diarrhoea (2 of 9). Oral mucosa ulcerations were present in 3 of 9 patients, and 1 patient presented gum mucosa hypertrophy. Signs and symptoms of gastrointestinal involvement are shown in Table III. In 5 patients, symptoms of gastrointestinal tract involvement occurred before the beginning of immunosuppressive therapy, in 1 case just after the induction of immunosuppressive therapy, and in the remaining 3 cases - from 1 to 12 months after. Two of 9 patients required surgical intervention. In 1 of them gastrointestinal perforation was found. Patient 2, with an established diagnosis of GPA, required immediate laparotomy due to positive peritoneal signs, acute abdominal pain, raised temperature, and diarrhoea. Seven months prior to the admission, the patient had finished a 9-month course of cyclophosphamide therapy and at that time was receiving a maintenance treatment with

Table I. Clinical characteristics of patients with GPA and gastrointestinal tract involvement

\begin{tabular}{|c|c|c|c|c|c|c|}
\hline Patient & Age & Gender & $\begin{array}{l}\text { Upper respiratory } \\
\text { tract involvement }\end{array}$ & $\begin{array}{l}\text { Pulmonary } \\
\text { involvement }\end{array}$ & $\begin{array}{c}\text { Renal } \\
\text { involvement }\end{array}$ & Other organs involvement \\
\hline 1 & 40 & M & + & + & + & Arthralgia, skin lesions, palsy of n. VII \\
\hline 2 & 74 & $\mathrm{~F}$ & + & + & + & Eye \\
\hline 3 & 50 & $\mathrm{~F}$ & + & + & + & Arthralgia, fever, skin lesions, CNS \\
\hline 4 & 54 & $\mathrm{~F}$ & + & - & - & None \\
\hline 5 & 18 & $\mathrm{~F}$ & + & + & + & Arthralgia \\
\hline 6 & 55 & $\mathrm{~F}$ & + & + & + & Arthralgia, fever, CNS, eye \\
\hline 7 & 59 & $\mathrm{~F}$ & + & + & + & Skin lesions \\
\hline 8 & 26 & $M$ & + & - & - & Parotid gland tumour \\
\hline 9 & 26 & $F$ & + & + & + & Arthralgia, skin lesions \\
\hline
\end{tabular}

CNS - central nervous system 
Table II. Results of laboratory and histopathological examinations

\begin{tabular}{|c|c|c|c|c|c|c|c|c|}
\hline Patient & $\begin{array}{c}\mathrm{Hb} \\
{[\mathrm{g} / \mathrm{dl}]}\end{array}$ & $\begin{array}{l}\text { WBC } \\
{[\mathrm{G} / \mathrm{I}]}\end{array}$ & $\begin{array}{l}\text { PLT } \\
{[\mathrm{G} / \mathrm{l}]}\end{array}$ & $\begin{array}{c}\text { CRP } \\
{[\mathrm{mg} / \mathrm{ll}]}\end{array}$ & $\begin{array}{l}\text { Creatinine } \\
\text { [mg/dl] }\end{array}$ & $\begin{array}{l}\text { CANCA } \\
{[\mathrm{RU} / \mathrm{ml}]}\end{array}$ & $\begin{array}{l}\text { Anti-PR3 } \\
{[\mathrm{RU} / \mathrm{ml}]}\end{array}$ & $\begin{array}{c}\text { Histopathological examination } \\
\text { of biopsy }\end{array}$ \\
\hline 1 & 7 & 14.9 & 736 & 115 & 7.8 & 320 & 30.5 & Kidney \\
\hline 2 & 9.5 & 20 & 366 & $n$ & 3.0 & 640 & 30 & Kidney \\
\hline 3 & 5.8 & 20.1 & 831 & $n$ & 10.5 & 1280 & 307 & Bronchus \\
\hline 4 & 6.6 & 5.4 & 324 & 8.8 & 0.9 & Negative & Negative & Nasal cavity tumour \\
\hline 5 & u & u & u & u & 7.9 & 160 & $n$ & Autopsy \\
\hline 6 & 8.5 & 16.08 & 765 & 304 & 1.9 & 320 & 118.5 & Pulmonis \\
\hline 7 & 6.2 & 7.2 & 401 & 24.8 & $\mathrm{HD}$ & 124 & $n$ & Nose \\
\hline 8 & 15.4 & 7.5 & 484 & 57.8 & 0.98 & 160 & 90 & Parotid gland tumour \\
\hline 9 & 15.5 & 7.6 & 403 & 81 & 0.7 & 640 & 299 & No confirmation \\
\hline
\end{tabular}

$n$-not performed, u-unknown, anti-PR3 - anti-proteinase 3 antibody, HD - haemodialysis.

Table III. Signs and symptoms of gastrointestinal tract involvement

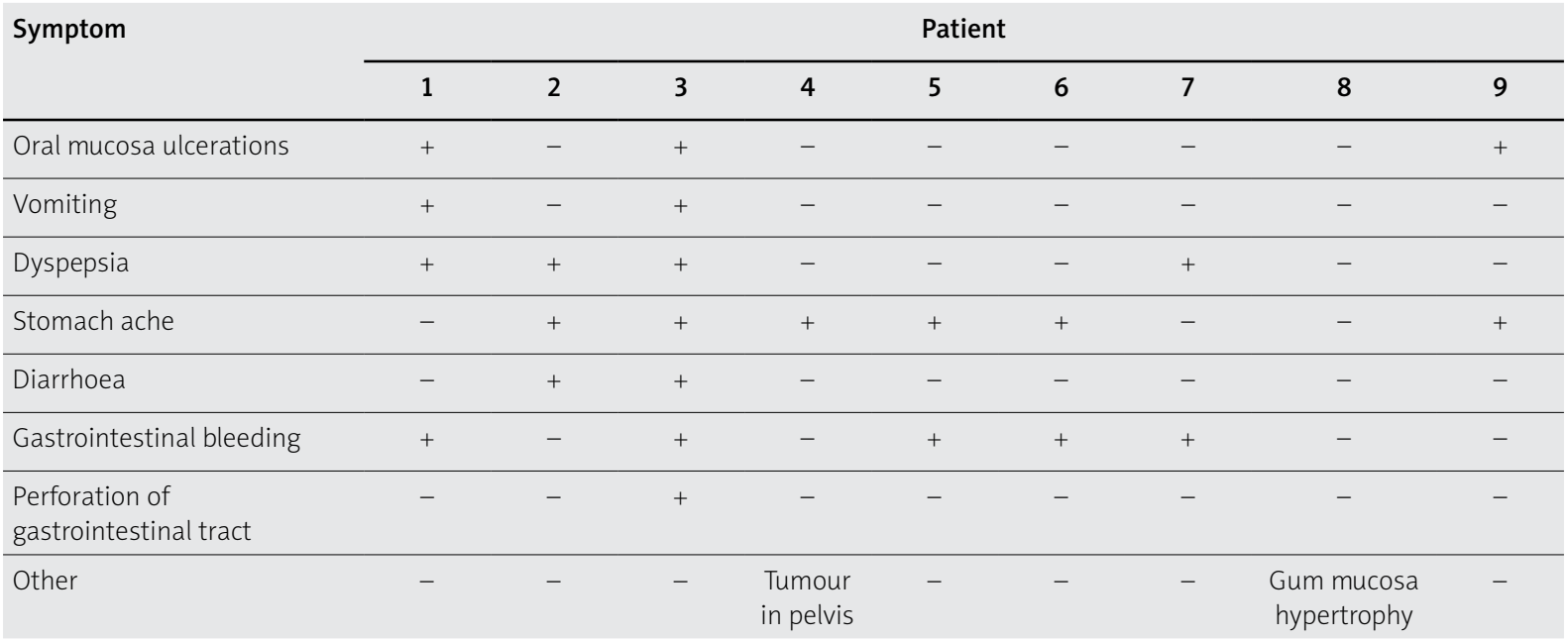

prednisone $20 \mathrm{mg} /$ day. The operation, however, did not prove any abdominal pathology. The symptoms persisted despite the wide-spectrum antibiotic therapy introduced afterwards. The high immune activity of the disease supports the opinion that the symptoms are related to GPA. Immunosuppressive treatment with methylprednisolone followed by prednisone orally and cyclophosphamide was introduced. Complete remission of symptoms was achieved.

Patient 4 was investigated due to severe abdominal pain and symptoms of upper respiratory tract involvement. An ultrasound study of the abdomen showed a partly cystic and partly solid tumour located behind the uterus. The patient was qualified to obstetric operation in 2 months. Simultaneously GPA was diagnosed by histopathological examination of a nasal cavity tumour specimen. Immunosuppressive treatment with prednisone (1 $\mathrm{mg} / \mathrm{kg}$ orally) and cyclophosphamide (800 mg IV once a month) was introduced. After 2 months an ultrasound examination performed before the planned operation showed no sign of the previously observed tumour in the pelvis. Involution of the tumour in response to immunosuppressive treatment suggests its relation to GPA. However, the aetiology of the tumour remains unclear, and the lack of a histopathological examination makes a final diagnosis impossible.

The second most common symptom in the presented group was gastrointestinal bleeding (in 5 patients). In the case of 3 of the patients the bleeding was severe, and caused death of 2 of them (patient 5 and 7). In the first of them bleeding was one of the initial symptoms, whereas in the other patient it accompanied a relapse of GPA during a course of immunosuppressive treatment with prednisone and cyclophosphamide. The au- 
topsy of patient 5 showed numerous deep ulcerations reaching the longitudinal muscle layer of the gastrointestinal wall located in the stomach, ileum, and colon; an autopsy of patient 7 was not performed. Patient 3, who also had severe gastrointestinal bleeding, required surgical intervention and removal of the terminal ileum, caecum, and sigmoid colon. Pathological examination proved inflammatory process and ulcers in the stomach, ileum, and colon, but no granulomas characteristic for GPA were found. The 2 remaining patients with less severe bleeding (patient 1 and 6) were successfully treated with proton-pump inhibitors. Gastroscopy in those individuals showed erosive gastritis and duodenitis. In both individuals colonoscopy was negative. The patients were receiving immunosuppressive treatment with prednisone that was intensified at the moment of aggravation of the disease by adding pulses of methylprednisolone and intravenous cyclophosphamide once a month. Additionally patient 6 underwent a procedure of plasmapheresis three times. The treatment was successful and a stop of gastrointestinal bleeding, improvement of renal function, and lung changes regression were achieved. Endoscopic examinations did not show any changes characteristic for GPA (granulomas or vasculitis). The characteristic features were found in resected ileum in patient 3 , and in an autopsy of patient 5 .

\section{Discussion}

Granulomatosis with polyangiitis is characterised by necrotising vasculitis and granulomatous inflammation. The disease has a predilection to the upper and lower respiratory tract and the kidneys, but most patients also show systemic disease. Gastrointestinal tract involvement is less common, effecting $10 \%$ to $24 \%$ of patients with GPA, and is usually detected in autopsy [4]. Vasculitis can cause local or diffuse pathologic changes in the gastrointestinal tract. The variety of lesions includes ulcer, submucosal oedema, haemorrhage, paralytic ileus, mesenteric ischaemia, bowel obstruction, and life-threatening perforation. The diagnosis of gastrointestinal complications depends largely on clinical manifestation. In the analysed group of patients, 9 of them (26\%) had gastrointestinal manifestations. Storesund et al. observed that gastrointestinal involvement is most frequently revealed in the first 2 years after the diagnosis of GPA [5], whereas all our patients manifested symptoms of gastrointestinal involvement within the first year. Moreover in 5 of them it presented as the initial symptom of GPA.

Pagnoux et al. in their group of 62 patients with systemic small and medium-sized vessel vasculitides and gastrointestinal tract involvement found, that the most frequent symptoms were abdominal pain (97\%), nausea or vomiting (34\%), diarrhea (27\%), hematochezia or melena (16\%), and hematemesis (6\%) [6]. The above symptoms were also observed by other investigators [7-10]. The location of pathological changes in the gastrointestinal tract is diverse and every part of the tract can be affected. Usually the pathological changes are located in the jejunum, ileum, stomach, and oesophagus. The most frequently described gross pathologies are ulcerations, intestinal wall necrosis, and perforations [4, 7, 11-25]. The gastrointestinal manifestation can be the first symptom of GPA that predicts involvement of other, more typical for the disease, organs [14]. Specimens taken during endoscopic examinations most often show uncharacteristic inflammation and ulcerations but very rarely confirm GPA [5, $7,15,16,20,22,26]$. Camilleri et al. suggested that this may be a result of biopsy taken too superficially during the endoscopy procedures [8], because the small and medium diameter vessels (typically involved in GPA) are located deeper in the submucosa. Besides, the pathologies are often located in regions difficult to access in a routine examination, such as appendix, gall bladder, and caecum [27]. In the analysed material histopathological confirmation of the specificity change was possible only in 2 of 9 cases (22\%) (in resected colon, and in autopsy - in ulcerations of the stomach, ileum, and colon). In a group of 62 patients with systemic small and medium-sized vessel vasculitides and gastrointestinal tract involvement reported by Pagnoux et al. [6], gastroduodenal ulcerations were detected endoscopically in 17 (27\%) patients, oesophageal in $7(11 \%)$, and colorectal in $6(10 \%)$, but histologic signs of vasculitis were found in only 3 colon biopsies. The differential diagnosis of gastrointestinal symptoms is difficult. The complaints can be associated with GPA, as well as with infectious and nonspecific inflammatory diseases of intestines, e.g. Crohn's disease. Crohn's disease shows a similarity of symptoms: involvement of gastrointestinal tract, eye, skin, and joints can be observed. In such a situation the final diagnosis is based on immunologic tests. pANCA are rarely present in patients with Crohn's disease (2-25\%), whereas cANCA are characteristically found in most cases of GPA [28]. In the literature, there are confounding reports about intestinal involvement in GPA. It is suggested that the use of corticosteroid therapy may be responsible for the development of intestinal manifestations. Therefore, in cases of gastrointestinal tract involvement that follows immunosuppressive treatment, it is essential to establish whether this is a result of a primary disease or a side effect of the therapy. Storesund argues that the gastrointestinal symptoms in patients with GPA are caused by a primary disease because the continuation 
of immunosuppressive therapy leads to remission [5]. It can be assumed that the immunosuppressive treatment should be given irrespectively of gastrointestinal symptoms. However, a careful control of the therapy is necessary. After induction therapy, cyclophosphamide should be changed to less toxic azathioprine or mycophenolate mofetil [29]. In cases of tract perforation, cholecystitis, or persistent gastrointestinal bleeding surgical intervention is necessary [7].

The authors of this paper are fully aware of the limitations of the present material, directly resulting from a lack of full pathologic confirmation of the origin of observed gastrointestinal abnormalities. Thus, the registered and analysed symptoms and signs could not be ascribed with absolute certainty to the GPA. As discussed above, due to often distant localisation of process and/or limited diagnostic possibilities of standard endoscopic procedures, confirmation of the specificity of changes is difficult (achieved only in 2 cases in our group). Nevertheless, the temporal relationship of the gastrointestinal symptoms and improvement after immunosuppressive treatment provide the basis for qualification of the discussed symptoms as related to GPA.

\section{Conclusions}

Active inflammatory process located in the gastrointestinal tract in the course of GPA is a rare complication; however, it does occur, causing a severe threat to the lives of patients. The differential diagnosis must cover other inflammatory diseases, especially Crohn's disease. The side effects of immunosuppressive treatment as a cause of intestinal complications must also be taken into consideration. Therapeutic strategies widely accepted for GPA treatment are effective in treating patients with gastrointestinal involvement in the course of the disease, although some complications require surgical intervention.

\section{Conflict of interest}

The authors declare no conflict of interest.

\section{References}

1. Jennette JC. Nomenclature and classification of vasculitis: les sons learned from granulomatosis with polyangiitis. Clin Exp Immunol 2011; 164 Suppl. 1: 7-10.

2. Wegener F. Uber generalisierte, septische Gefasserkrankungen. Vert Dtsch Ges Pathol 1936; 29: 202-6.

3. Basu N, Watts R, Bajema I, et al. EULAR points to consider in the development of classification and diagnostic criteria in systemic vasculitis. Ann Rheum Dis 2010; 69: 1744-50.

4. Sahin M, Cure E, Goren I, et al. Wegener's granulomatosis presenting with acute renal failure and gastric ulcer. Case Rep Clin Prac Rev 2006; 7: 236-9.
5. Storesund B, Gran JT, Koldingsnes W. Severe intestinal involvement in Wegener's granulomatosis: report of two cases and review of the literature. Br J Rheumatol 1998; 37: 387-90.

6. Pagnoux C, Mahr A, Cohen P, et al. Presentation and outcome of gastrointestinal involvement in systemic necrotizing vasculitides: analysis of 62 patients with polyarteritis nodosa, microscopic Polyangiitis, Wegener granulomatosis, Churg-Strauss syndrome or rheumatoid arthritis-associated vasculitis [abstract]. Medicine (Baltimore) 2005; 84: 115-28.

7. Haworth S, Pusey C. Severe intestinal involvement in Wegener's granulomatosis. Gut 1984; 25: 1296-300.

8. Camilleri M, Pusey C, Rees A. Gastrointestinal manifestations of systemic vasculitis. QJM 1983; 52: 141-9.

9. Haworth S. Renal involvement in Wegener's granulomatosis. The Hammersmith experience. In: Nephrology'83. D'Amico G (ed.). Wichtig Editore, Milan 1983.

10. Fauci A, Haynes B, Katz P. Wegener's granulomatosis: prospective clinical and therapeutic experience with patients for 21 years. Ann Intern Med 1983; 98: 76-85.

11. Akca T, Colak T, Caglikulekci M, et al. Intestinal perforation in Wegener's granulomatosis: a case report. Ulus Travma Derg 2005; 11: 348-51.

12. Geraghty J, Mackay IR, Smith D. Intestinal perforation in Wegener's granulomatosis. Gut 1986; 27: 450-1.

13. Kitamura N, Matsukawa Y, Takei M, et al. Wegener's granulomatosis complicated with intestinal ulceration. Mod Rheumatol 2004; 14: 480-4.

14. Schneider A, Menzel J, Gaubitz M, et al. Colitis as the initial presentations of Wegener's granulomatosis. J Intern Med 1997; 242: 513-7.

15. Deniz K, Özseker H S, Balas S, et al. Intestinal involvement in Wegener's granulomatosis. J Gastrointestin Liver Dis 2007; 16: 329-31.

16. Coward R, Gibbons C, Brown C, et al. Gastrointestinal haemorrhage complicating Wegener's granulomatosis. BMJ 1985; 291: 865-6.

17. Umehara Y, Kudo M, Minami Y, et al. Wegener's granulomatosis complicated with aphthoid colitis [abstract]. Dig Endosc 2006; 18: 221-4.

18. Srinivasan U, Coughlan RI. Small intestinal perforation complicating Wegener's granulomatosis. Rheumatology 1999; 38: 289-90.

19. McNabb W, Lennox M, Wedzicha J. Small intestinal perforation in Wegener's granulomatosis. Postgrad Med J 1982; 58: 123-5.

20. Shaikh F, Sabu C, Peirce T, et al. Extensive intestinal ischaemic necrosis in Wegener's granulomatosis. Gut 2006; 55: 1368-9.

21. Pickhardt P, Curran V. Fulminant enterocolitis in Wegener's granulomatosis: CT findings with pathologic correlation. Am J Roentgenol 2001; 177: 1335-7.

22. Deger S, Sahin T, Vural C, et al. Wegener's granulomatosis with massive gastrointestinal hemorrhage due to jejunal and colonic involvement: report of a case. Surg Today 2008; 38: 166-9.

23. Tokuda M, Kurata N, Daikkuhara H, et al. Small intestinal perforation in Wegener's granulomatosis [abstract]. J Rheumatol 1998; 16: 547-9.

24. Fallow G, Hamilton S, Taylor D, et al. Esophageal involvement in Wegener's granulomatosis: a case report and review of the literature. Can J Gastroenterol 2000; 14: 449-51. 
25. Zdrojewski Z, Masiak A, Bułło B, et al. Renal involvement in Wegener's granulomatosis - clinical course and outcome. Ann Acad Med Gedan 2005; 35 Suppl. 10: 53-8.

26. Bułto B, Manitius J, Rutkowski B, et al. Ziarniniak Wegenera z zajęciem nerek - obserwacje własne. Nefrol Dializoter Pol 1999; 3: 293-6.

27. Garcia-Porrua C, Gutierrez-Duque O, Soto S, et al. Localized vasculitis of the gastrointestinal tract. Semin Arthritis Rheum 2006; 35: 403-6.

28. Temmesfeld-Wollbrueck B, Heinrichs C, Szalay A, et al. Granulomatous gastritis in Wegener's disease: differentiation from Crohn's disease supported by a positive test for antineutrophil antibodies. Gut 1997; 40: 550-3

29. Swarowska-Knap J, Tłustochowicz W. Praktyczne aspekty leczenia układowych zapaleń naczyń. Reumatologia 2007; 45: 278-83.

Received: 24.11.2014

Accepted: 17.06.2015 